\title{
DÉSORDRE CORRÉĹÉ: UN DEGRÉ DE LIBERTÉ POUR LE CONTRÔLE
DES PROPRIÉTÉS PHOTONIQUES
}

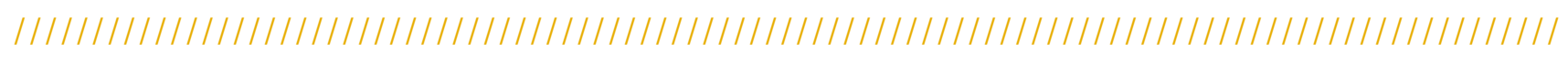
Rémi CARMINATI ' et Romain PIERRAT ${ }^{\dagger}$ Institut Langevin, ESPCI Paris, Université PSL, CNRS, Paris, France *remi.carminati@espci.psl.eu, ${ }^{\dagger}$ romain.pierrat@espci.psl.eu

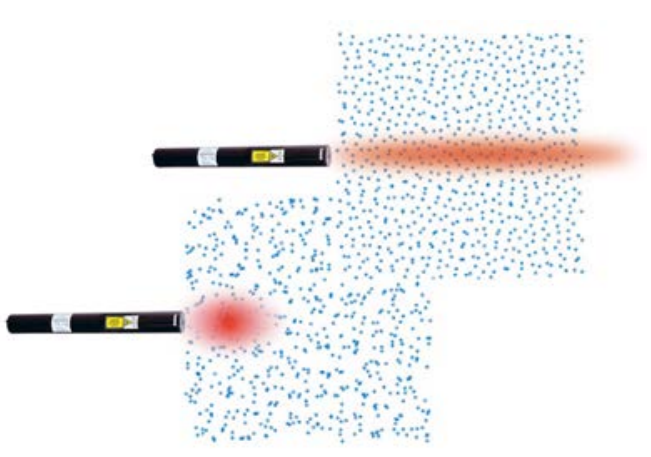

https://doi.org/10.1051/photon/202111147
L'ordre partiel dans un matériau désordonné peut modifier son aptitude à diffuser la lumière. Les propriétés photoniques de tels matériaux sont ainsi fortement influencées par le niveau d'ordre partiel, décrit par les corrélations statistiques entre les positions des centres diffuseurs. Générer de telles corrélations offre un degré de liberté pour la conception de matériaux désordonnés aux propriétés innovantes.

Article publié en accès libre sous les conditions définies par la licence Creative Commons Attribution License CC-BY (https://creativecommons.org/licenses/by/4.0), qui autorise sans restrictions l'utilisation, la diffusion, et la reproduction sur quelque support que ce soit, sous réserve de citation correcte de la publication originale.

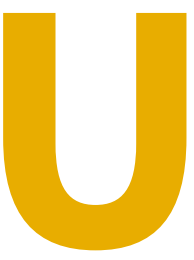

$\mathrm{n}$ matériau désordonné à l'échelle de la longueur d'onde diffuse la lumière. La forte réflectivité d'une peinture blanche ou d'une feuille de papier, la couleur bleue du ciel, l'opacité d'un brouillard ou la coloration de certains tissus biologiques sont différentes manifestations de la diffusion de la lumière. Un paramètre clé pour caractériser le niveau de diffusion est le libre parcours moyen de diffusion, noté $\ell_{s}$ et qui représente la distance moyenne parcourue par la lumière entre deux événements de diffusion successifs. Lorsque $\ell_{s}$ est très grand par rapport à la taille caractéristique $L$ du milieu diffusant, la lumière traverse sans être diffusée (régime dit balistique). À l'inverse, lorsque $\ell_{s}$ est très inférieur à $L$, la lumière est diffusée fortement (régime de diffusion multiple). Pour fixer les idées, le libre parcours moyen pour la lumière visible est de l'ordre de la dizaine de micromètres dans une peinture blanche, du micromètre dans une poudre très compacte, de la centaine de micromètres dans les tissus biologiques dans le proche infrarouge (longueurs d'ondes utilisées en optique biomédicale).

Considérons un matériau diffusant formé de diffuseurs dispersés dans une matrice continue (par exemple des particules colloïdales en suspension dans un liquide, des billes micrométriques compactées sous forme de poudre, un nuage, ou un modèle simpliste de tissu biologique). Dans la vision la plus simple, on peut considérer que les positions des centres diffuseurs sont statistiquement indépendantes. Le matériau est alors totalement désordonné. Si cette vision suffit à comprendre les propriétés optiques de matériaux dilués, elle est mise en défaut lorsque la concentration en diffuseurs augmente suffisamment pour que les interactions de contact ne soient plus négligeables. D'autres interactions à plus longue portée (par exemple électrostatiques ou hydrodynamiques) peuvent également favoriser l'apparition de corrélations statistiques dans les positions des diffuseurs. La présence de ces corrélations change sensiblement le libre parcours moyen de diffusion, et donc les propriétés photoniques des matériaux diffusants. Même $\bullet \circ$ 
si ce mécanisme est connu depuis longtemps, la possibilité de contrôler, dans une certaine mesure, la formation d'un désordre corrélé permet d'imaginer aujourd'hui de nouvelles approches pour la conception de matériaux photoniques [1].

\section{TRANSPARENCE D'UN MATÉRIAU BIOMIMÉTIQUE DÉSORDONNÉ}

En premier lieu, examinons un exemple concret. Partant d'une solution de collagène moléculaire dans l'eau, il est possible de former un matériau prenant la forme d'un gel dont les propriétés mécaniques et optiques sont pilotées par une sous-structure formée d'un réseau complexe de fibrilles de collagène (voir figure 1, issue des travaux de l'équipe de Nadine Nassif au Laboratoire de Chimie de la Matière Condensée, Sorbonne Université, Collège de France, CNRS). Cette structuration résulte d'un processus d'auto-organisation que nous ne décrirons pas ici [2]. Le résultat important pour nous est que le réseau de fibrilles est désordonné, rendant le matériau diffusant, blanc et opaque pour une épaisseur de quelques millimètres. Pourtant, au voisinage d'une concentration particulière en collagène, il apparait une zone de transparence, visible sur la photographie au centre de la figure 1 . De part et d'autre de cette zone de transparence, c'est-à-dire à plus forte ou plus basse concentration en collagène, le matériau est beaucoup plus diffusant, comme attesté par des mesures du libre parcours moyen qui chute de part et d'autre de la zone de transparence. Ce caractère non monotone $\mathrm{du}$ niveau de diffusion lorsque la concentration en collagène change montre que l'émergence de transparence n'est pas un simple effet de dilution (qui persisterait à concentration encore plus faible). Elle ne s'explique que par l'apparition d'une structuration du réseau de fibrilles de collagène présentant de l'ordre partiel permettant de réduire la diffusion de la lumière au voisinage d'une concentration spécifique en collagène [2].

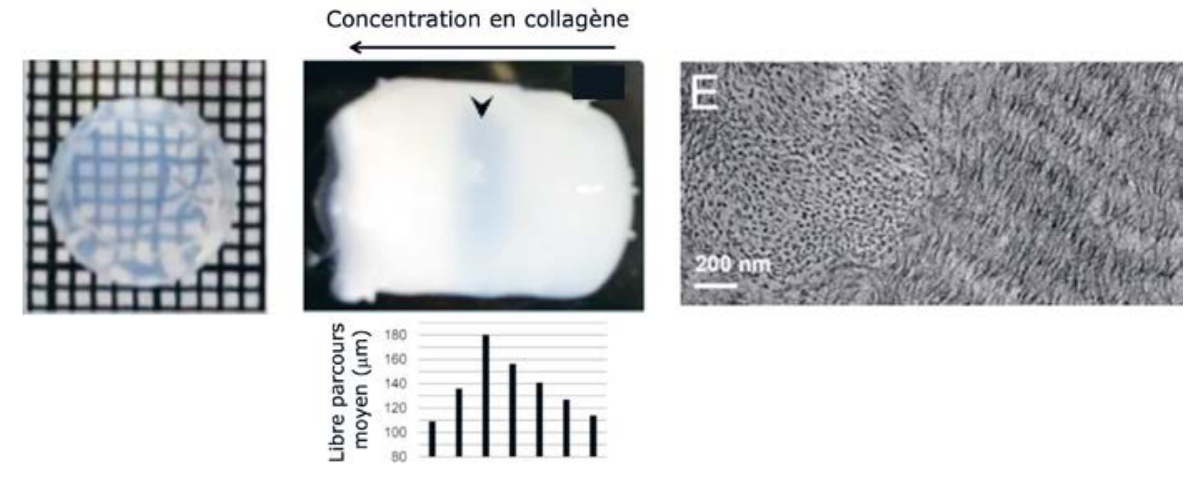

Figure 1. Gauche: matériau formé par l'auto-organisation de fibrilles de collagène dans l'eau, prenant la forme d'un gel, et transparent pour une concentration critique en collagène moléculaire initial. La taille de l'objet est de $1 \mathrm{~cm}$. Centre : matériau présentant un gradient de concentration en collagène. La bande de transparence, autour de la concentration critique, est indiquée par la flèche. Le libre parcours moyen mesuré dans les différentes zones montre un accroissement notable dans la zone de transparence. Droite : image TEM montrant la structure microscopique du réseau de fibrilles de collagène, et l'organisation typique à la concentration critique. Adaptée de [2] avec autorisation.

Lorsque cet ordre partiel est modifié ou perdu, le matériau redevient diffusant. Notons que ce mécanisme est similaire à celui expliquant la transparence de la cornée, dont ces hydrogels de collagène sont un bon modèle artificiel biomimétique.

\section{DÉSORDRE CORRÉLÉ : DIFFUSION, TRANSPARENCE \\ ET ABSORPTION}

La structure des hydrogels de collagène discutés ci-dessus est trop complexe pour qu'un lien direct simple entre le libre parcours moyen de diffusion et la microstructure puisse être établi. Il est cependant possible d'illustrer l'apparition de transparence sur un modèle simplifié. Considérons un matériau formé de petits diffuseurs sphériques identiques distribués aléatoirement dans une matrice transparente. Lorsque les positions des diffuseurs sont indépendantes, le libre-parcours moyen de diffusion suit la loi simple:

$$
\frac{1}{\ell_{s}}=\rho \sigma_{s}
$$

avec $\rho$ la densité volumique de diffuseurs (nombre par unité de volume) et $\sigma_{s}$ la section efficace de diffusion d'un diffuseur individuel. En présence de désordre corrélé, l'expression du libre parcours moyen doit être modifiée.
Elle dépend du facteur de structure $S(q)$ qui décrit les corrélations statistiques des positions de deux diffuseurs différents, et module l'intensité diffusée par chaque élément de volume (voir encadré). En présence de corrélations du désordre, le libre parcours moyen s'écrit [3] :

$$
\frac{1}{\ell_{s}}=\frac{2 \pi \rho}{k_{\mathrm{r}}^{4}} \int_{0}^{2 \mathrm{k}_{\mathrm{r}}} q F(q) S(q) d q .
$$

Ici $k_{\mathrm{r}}=2 \pi / \lambda$ où $\lambda$ est la longueur d'onde dans le milieu environnant et $F(q)$ caractérise la diffusion par un diffuseur isolé (l'intégrale de $F(q)$ est proportionnelle à $\sigma_{\mathrm{s}}$ ). À priori, la présence de corrélations peut conduire à une diminution ou à une augmentation du libre parcours moyen. Qualitativement, si $S(q)<<1$ pour $q \leq 2 k_{\mathrm{r}}$ on s'attend à une augmentation du libre parcours moyen $\ell_{s}$ par rapport à un matériau formé des mêmes diffuseurs, à même densité, mais positionnés de manière complètement aléatoire (dans ce cas $S(q)=1$ ). C'est un mécanisme de ce type qui est responsable de la transparence des hydrogels de collagène discutés précédemment. Dans un cas plus extrême pour lequel $S(q)$ s'annule au voisinage de $q=0$, l'effet peut être très spectaculaire. Un désordre corrélé de ce type est dit hyperuniforme (voir 
encadré). Les structures hyperuniformes ont eu un intérêt croissant ces dernières années dans des domaines allant de la matière molle à la physique des ondes [4].

Une illustration de l'effet de l'hyperuniformité par des simulations numériques est présentée sur la figure 2, qui compare la distribution d'intensité diffusée par une structure totalement désordonnée (à gauche) et par une structure hyperuniforme formée des mêmes diffuseurs à même densité (au centre). Dans cet exemple, le matériau désordonné présente un libre parcours moyen inférieur à son épaisseur et est donc opaque, avec une forte intensité réfléchie caractéristique de la diffusion multiple. En revanche, pour le matériau hyperuniforme, l'intensité diffusée est quasi nulle. Le libre parcours moyen est devenu très grand devant l'épaisseur du milieu qui se comporte alors comme un matériau transparent [5]. Spectralement, ce phénomène s'observe pour les longueurs d'onde suffisamment grandes pour que $S(q)$ s'annule dans le domaine d'intégration de l'expression de $\ell_{\mathrm{s}}$ ci-dessus. Au-delà de la réduction du niveau de diffusion, les matériaux hyperuniformes peuvent également avoir un intérêt

\section{ORDRE PARTIEL ET FACTEUR DE STRUCTURE}

Le facteur de structure permet de décrire et de mesurer le niveau d'ordre partiel d'une structure désordonnée. Pour un système de $N$ points, de positions $r_{\mathrm{i}}$, il est défini comme

$$
S(q)=\frac{1}{N}\left\langle\left|\sum_{j=1}^{N} e^{i q \cdot r_{j}}\right|^{2}\right\rangle
$$

où $\langle\ldots\rangle$ désigne une moyenne statistique sur l'ensemble des réalisations du désordre (positions des points). Pour un système statistiquement isotrope, le facteur de structure ne dépend que de $q=|q|$. En développant l'expression, on verrait apparaître des termes croisés faisant intervenir deux points différents et ne s'annulant pas en présence de corrélations. Si les points représentent les positions des centres diffuseurs d'un matériau, $S(q)$ décrit les interférences entre les ondes diffusées par deux diffuseurs différents. Pour chaque élément de volume du matériau, l'intensité diffusée dans une direction $\boldsymbol{k}_{s}$ pour une onde incidente dans la direction $\boldsymbol{k}_{i}$ est proportionnelle à $S(q)$ calculé pour $\boldsymbol{q}=\boldsymbol{k}_{s}-\boldsymbol{k}_{i}$. La figure cicontre (adaptée de [1], produite par K. Vynck) montre deux distributions de points présentant un ordre partiel ainsi que leurs facteurs de structure. À gauche, l'ordre est à courte portée et le facteur de structure a une forme similaire à celui observé pour les molécules d'un liquide (pour un milieu totalement désordonné, il serait constant et égal à un, comme pour les molécules d'un gaz parfait). À droite, la distribution de points suit un ordre partiel dit hyperuniforme, pour lequel le facteur de structure s'annule au voisinage de l'origine.
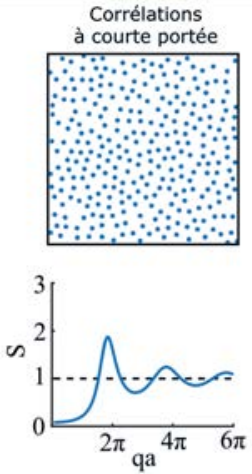

Hyperuniformité
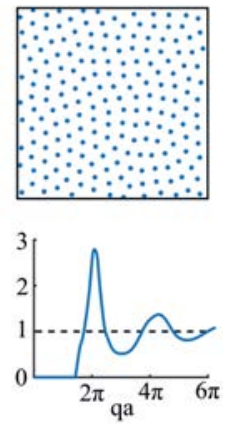

\section{SPECTROGON}

Filtres Interférentiels

De 200 á 15000 nm

- Passe-bande

- Passe-haut

- Passe-bas

- Large bande

- Densité neutre

- Disponible en stock

\section{Réseaux Holographiques}

De 150 á 2000 nm

- Compression d'impulsion

- Télècom

- Accordabilité spectrale

- Monochromateurs

- Spectroscopie

- Disponible en stock
UK (parle francais): sales.uk@spectrogon.com·Tel +44 1592770000 Sweden (headquarters): sales se@spectrogon com • Tel +4686382800 US: sales.us@spectrogon.com·Tel +19733311191 

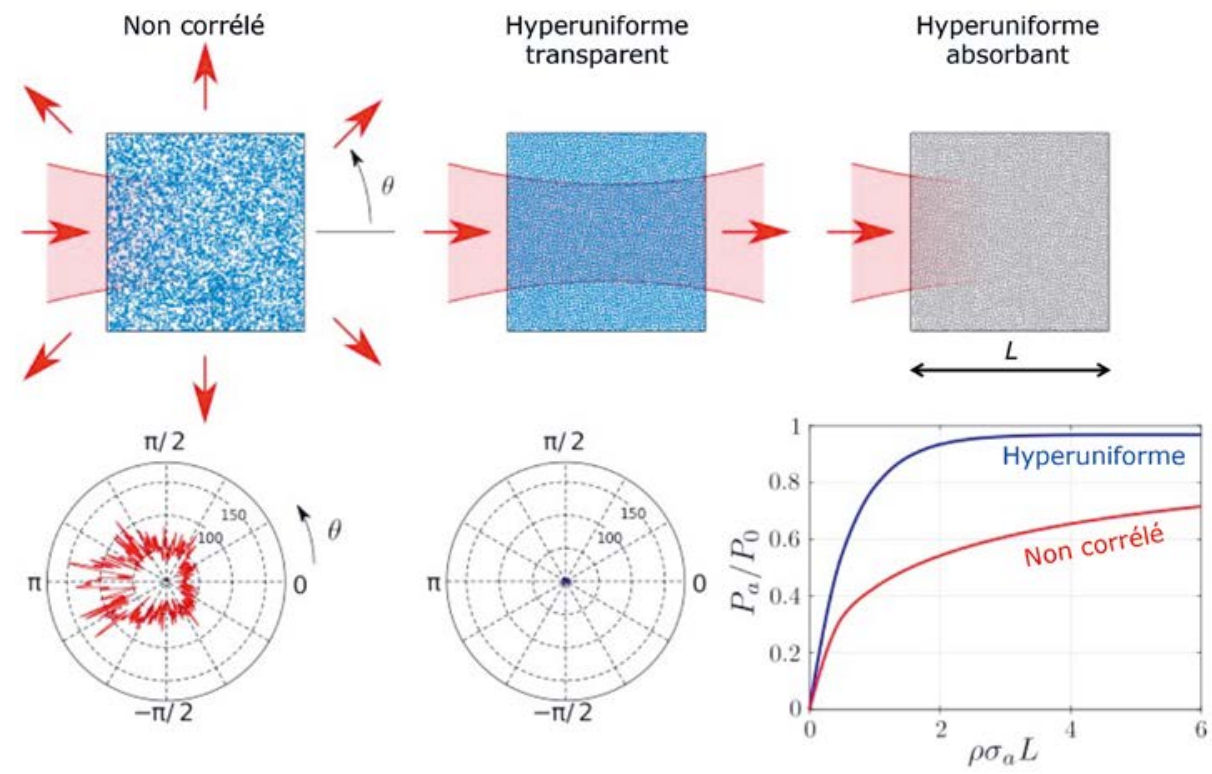

Figure 2. Simulations numériques de la transparence ou de l'absorption induite par des corrélations spatiales du désordre (ici dans le cas particulier du désordre hyperuniforme). Gauche : dans le cas d'un matériau formé de diffuseurs identiques non corrélés, et d'épaisseur grande devant $\ell_{s}$, on observe un fort niveau de diffusion, comme indiqué par le diagramme de diffusion du bas (intensité diffusée en fonction de l'angle d'observation). Centre : dans un matériau hyperuniforme de même densité, le niveau de diffusion est fortement réduit. Le matériau apparait transparent, comme un matériau homogène. Droite : si les diffuseurs sont absorbants, la réduction de la diffusion favorise l'absorption. La courbe du bas montre la puissance moyenne absorbée (normalisée par la puissance incidente) lorsque la section efficace d'absorption $\sigma_{a}$ d'un diffuseur individuel augmente. L'absorption est maximisée pour la structure hyperuniforme qui minimise la diffusion. Adaptée de [5] et [6] avec autorisation.

pour former des absorbeurs peu denses, constitués de particules absorbantes distribuées dans une matrice transparente. En effet, en réduisant la diffusion par le matériau, on peut maximiser l'absorption. Cette possibilité est illustrée sur la figure 2 (à droite), où l'on compare la puissance moyenne absorbée par un matériau totalement désordonné (courbe rouge) et par un matériau hyperuniforme formé des mêmes particules à même densité (courbe bleue). On observe que la puissance absorbée peut être maximisée en utilisant un désordre corrélé hyperuniforme [6]. Le système étant désordonné et non résonant, cette maximisation s'observe sur une large bande spectrale et une grande plage d'angles d'incidence, rendant le matériau proche d'un corps noir. Le mécanisme sous-jacent est relativement simple. En réduisant la diffusion, l'hyperuniformité crée un matériau effectif homogène pour la lumière incidente qui, si l'épaisseur

\section{RÉFÉRENCES} (Cambridge University Press, Cambridge, 2021), chap. 18

[4] S. Torquato, Phys. Rep. 745, 1 (2018)

[5] O. Leseur, R. Pierrat and R. Carminati, Optica 3, 763 (2016) R. Pierrat and R. Carminati, Phys. Rev. A 101, 053829 (2020) est suffisante, est absorbée progressivement sans ressortir du milieu.

\section{VERS UNE INGÉNIERIE DU DÉSORDRE}

Les matériaux à désordre corrélé, intermédiaires entre des matériaux totalement amorphes et des cristaux photoniques, présentent un grand potentiel pour le façonnage de propriétés optiques originales et à priori robustes aux imperfections. De plus, la possibilité de générer de l'ordre partiel par auto-organisation, qui est également un sujet d'étude dans le domaine de la matière molle, offre des perspectives pour des procédés permettant une fabrication à grande échelle. Au-delà des illustrations simples présentées ici, de nombreux phénomènes sont explorés, comme l'apparition de bandes photoniques interdites même en l'absence de périodicité ou la localisation d'Anderson [7]. Par ailleurs, certains types d'ordres partiels peuvent favoriser ou inhiber la diffusion à des longueurs d'ondes sélectionnées, permettant l'apparition de colorations structurelles en labsence de pigments absorbants [8]. Pour des études fondamentales ou la conception de matériaux innovants, l'exploitation du désordre corrélé est un degré de liberté prometteur qui reste encore largement à exploiter.

[1] K. Vynck, R. Pierrat, R. Carminati, L.S. Froufe-Pérez, F. Scheffold, R. Sapienza, S. Vignolini and J.J. Sáenz, Light in correlated disordered media, arXiv:2106.1389 (2021)

[2] C. Salameh, F. Salviat, E. Bessot et al., Proc. Natl. Acad. Sci. USA 117, 11947 (2020)

[3] R. Carminati and J.C. Schotland, Principles of Scattering and Transport of Light

[6] F. Bigourdan, R. Pierrat and R. Carminati, Opt. Express 27, 8666 (2019); A. Sheremet,

[7] J. Haberko, L.S. Froufe-Pérez and F. Scheffold, Nature Comm. 11, 4867 (2020) ; G.F. Aubry, L.S. FroufePérez, U. Kuhl, O. Legrand, F. Scheffold and F. Mortessagne, Phys. Rev. Lett. 125, 127402 (2020) [8] V.E. Johansen, O.D. Onelli, L.M. Steiner and S. Vignolini, Photonics in Nature: From Order to Disorder, in S. Gorb, E. Gorb (eds), Functional Surfaces in Biology III (Springer, Berlin, 2017) 